\title{
NIVELES DE PLOMO EN GESTANTES Y NEONATOS EN LA CIUDAD DE LA OROYA, PERÚ
}

\author{
Jorge Castro-Bedriñana1,a, Doris Chirinos-Peinado1,b, Elva Ríos-Ríos²,c
}

\section{RESUMEN}

\begin{abstract}
Objetivos. Determinar los niveles de plomo $(\mathrm{Pb})$ en gestantes y neonatos en la ciudad de La Oroya, Perú. Materiales $y$ métodos. Se realizó un estudio transversal. La población estuvo conformada por gestantes residentes durante al menos dos años en la ciudad de La Oroya, cuando operaban normalmente las fundiciones de Pb, cobre y zinc. Se obtuvo muestras de sangre de la gestante antes del parto y luego del parto se tomaron muestras de placenta y sangre del cordón umbilical. El nivel de $\mathrm{Pb}$ de dichas muestras fue evaluado por espectrometría de absorción atómica con horno de grafito. Se determinaron regresiones cuadráticas y correlaciones de Pearson. Resultados. Se evaluó cuarenta partos normales. Los niveles promedio de $\mathrm{Pb}$ en la sangre materna, cordon umbilical y placenta fueron $27,4 \pm 15,6 \mathrm{ug} / \mathrm{dL} ; 19,0 \pm 12,6 \mathrm{ug} / \mathrm{dL}$ y $319,0 \pm 215,9 \mathrm{ug} / 100 \mathrm{~g}$, respectivamente. El $67,5 \%$ de los neonatos tuvieron más de $10 \mathrm{ug} / \mathrm{dL}$ de $\mathrm{Pb}$. El nivel de $\mathrm{Pb}$ de la sangre del cordón umbilical representó el 69,4\% del nivel de $\mathrm{Pb}$ en sangre materna. Los coeficientes de correlación entre el $\mathrm{Pb}$ de la sangre materna y cordón umbilical, sangre materna y placenta, placenta y sangre del cordón umbilical fueron 0,$36 ; 0,48$ y 0,33 , respectivamente. Conclusiones. Cuando funcionaba la fundición, las gestantes y recién nacidos tenían concentraciones elevadas de $\mathrm{Pb}$ en sangre. Las concentraciones de $\mathrm{Pb}$ en la placenta y cordón umbilical tuvieron una correlación moderada con el Pb de la sangre materna. El Pb de la sangre materna y cordón umbilical fue 1,5 veces más alto que los de zonas más alejadas.
\end{abstract}

Palabras clave: Plomo; Mujeres embarazadas; Recién nacido; Cordón umbilical; Metalurgia (fuente: DeCS BIREME).

\section{LEAD LEVELS IN PREGNANT WOMEN AND NEWBORNS IN LA OROYA CITY, PERU}

\section{ABSTRACT}

Objectives. To determine blood-lead $(\mathrm{Pb})$ levels in pregnant women and neonates in a metallurgical city of Peru. Materials and methods. A cross-sectional study was carried out, the population of which consisted of pregnant women who had lived for at least two years in the city of La Oroya, at the time when the lead, copper and zinc foundries operated normally. A blood sample was obtained from the pregnant women before delivery and, after delivery, from the neonate's umbilical cord and placenta. Lead levels in those samples were measured by graphite furnace atomic absorption spectrophotometry. Pearson's squared regressions and correlations were determined. Results. Forty normal deliveries were studied. The average blood lead levels in the mother, umbilical cord and placenta were $27.4 \pm 15.6 \mathrm{ug} / \mathrm{dL} ; 19.0 \pm 12.6 \mathrm{ug} / \mathrm{dL}$ and $319.0 \pm 215.9 \mathrm{ug} / 100 \mathrm{~g}$, respectively. $67.5 \%$ of the neonates registered levels higher than $10 \mathrm{ug} / \mathrm{dL}$. Pb levels in the umbilical cord accounted for $69.4 \%$ of $\mathrm{Pb}$ level in maternal blood. The correlation coefficients between $\mathrm{Pb}$ levels in maternal blood and umbilical cord, maternal blood and placenta, and placenta and umbilical cord were $0.36 ; 0.48$ and 0.33 , respectively. Conclusions. At the time the foundry operated, pregnant women and newborns had high blood lead concentrations. Pb concentrations in the placenta and umbilical cord had moderate correlation with the $\mathrm{Pb}$ levels in maternal blood. $\mathrm{Pb}$ levels in maternal blood and umbilical cord were 1.5 times as high as in more distant areas.

Key words: Lead; Pregnant women; Infant, newborn; Umbilical cord; Metallurgy (source: MeSH NLM).

\section{INTRODUCCIÓN}

La emisión de metales pesados al ambiente, sobre todo en zonas de fundición, tiene un marcado efecto sobre la salud, en particular en la población infantil (1-5). La contaminación por plomo $(\mathrm{Pb})$ es una de las más peligrosas para el binomio madre-niño; pues, por el fácil tránsito trasplacentario del metal (6-11,), los recién nacidos estarían en mayor riesgo de sufrir retraso del crecimiento, daños neurológicos, psicomotores, cognitivos, metabólicos, entre otros; mientras que en las gestantes provoca abortos, partos prematuros y muerte prenatal (12-15). Por estas razones, el Centro de Control y Prevención de Enfermedades de los Estados Unidos (CDC) ${ }^{(16,17)}$ y la

\footnotetext{
Universidad Nacional del Centro del Perú. Huancayo, Perú.

Universidad Nacional Agraria La Molina. Lima, Perú.

Doctor en salud pública; ${ }^{\mathrm{b}}$ doctora en salud pública; ${ }^{\mathrm{c}}$ magister en nutrición.

Recibido: 09-04-13 Aprobado: 05-06-13
} 
Organización Mundial de la Salud (OMS) ${ }^{(18)}$, señalan como el límite crítico de $\mathrm{Pb}$ en la sangre un nivel de $10 \mathrm{ug} / \mathrm{dL}$, para las gestantes y para menores de cinco años.

EI CDC ha venido reportando una tendencia histórica sobre la reducción gradual de los niveles de $\mathrm{Pb}$ sanguíneo indicado como límites máximos, siendo para los años 1960, 1971, 1975, 1985, 1990 y 1991, de 60, 40, 30, 25, 15 y $10 \mathrm{ug} / \mathrm{dL}$, respectivamente. Por su parte, el Departamento de Salud de los Estados Unidos (19), indica que el deterioro de la función cognitiva empezaría por encima de los $10 \mathrm{ug} / \mathrm{dL}$.

Con respecto al contenido de $\mathrm{Pb}$ en la placenta, lyengar y Rapp ${ }^{(10,20,21)}$, indican que la placenta se expone a diferentes sustancias dañinas y en el caso de metales tóxicos, el tejido placentario puede ser un biomarcador dual, para evaluar la salud materna y fetal. El rango promedio de las concentraciones de $\mathrm{Pb}$ que determinaron en diferentes trabajos realizados en zonas de baja contaminación fue de 5-60 ng/g; observándose que la placenta parece ser una barrera débil para el $\mathrm{Pb}$. En Shanghai ${ }^{(6)}$, en 165 mujeres ocupacionalmente no expuestas al $\mathrm{Pb}$, informan que el tejido placentario tuvo un promedio de 17,85 ug/100 g.

Aun cuando un gran número de estudios demuestran la relación entre niveles de $\mathrm{Pb}$ en sangre materna, placenta y cordon umbilical en condiciones de baja contaminación, se hace necesario realizar investigaciones en zonas altoandinas con altos niveles de contaminación por $\mathrm{Pb}, \mathrm{y}$ otros metales pesados. En el Perú, la ciudad metalúrgica de La Oroya fue catalogada como la quinta ciudad más contaminada del planeta ${ }^{(22)}$. En La Oroya, desde el año 1922, la población ha estado expuesta a las emisiones de la planta de fundición. Estudios llevados a cabo por el Ministerio de Salud del Perú, indican que 99\% de los niños que viven en La Oroya y sus alrededores, han mostrado niveles que exceden las cantidades críticas hasta hoy aceptadas, indicando que aproximadamente unas 35000 personas están potencialmente afectadas por la contaminación de plomo en la zona.

El objetivo de la presente investigación fue determinar los niveles de $\mathrm{Pb}$ en la sangre materna y su relación sobre su concentración en la placenta y sangre del cordón umbilical, en una ciudad metalúrgica del Perú.

\section{MATERIALES Y MÉTODOS}

\section{TIPO Y LUGAR DE ESTUDIO}

Se realizó un estudio transversal en el Hospital II EsSalud de La Oroya ubicado en la provincia de Yauli, en el departamento de Junín a 3730 metros de altura, cuando operaba normalmente las fundiciones de cobre, zinc y $\mathrm{Pb}$ de la compañía Doe Run Perú (2003-2004). La ciudad de La Oroya tiene dos zonas poblacionales diferenciadas que son La Oroya Antigua (cercana a la zona de fundición) y La Oroya Nueva. Los análisis de Pb se realizaron en el Laboratorio de Análisis Quimico del Instituto Peruano de Energía Nuclear (IPEN).

\section{POBLACIÓN}

Gestantes atendidas en el Hospital II EsSalud de La Oroya, institución que brinda atención a más del $90 \%$ de los partos de dicha localidad. La asignación de los casos al grupo de estudio fue secuencial conforme se fueron presentando. Los criterios de inclusión consideraron: embarazo normoevolutivo; vivir al menos dos años en La Oroya, incluido su proceso de gestación; no presentar diabetes, hipertensión, insuficiencia renal, epilepsia, toxemia gravídica, infección de transmisión sexual, ni anormalidades genéticas; asi como no tener impedimento físico o mental para comunicarse y aceptar participar voluntariamente en el estudio.

\section{MUESTRAS BIOLÓGICAS YANÁLISIS QUÍMICO}

Para la colección de muestras hematológicas se utilizó tubos vacutainer de $10 \mathrm{~mL}$ con heparina. Previo al trabajo de parto se muestreó $10 \mathrm{~mL}$ de sangre materna y luego de la recepción del neonato se tomaron $10 \mathrm{~mL}$ de sangre del cordón umbilical con la placenta in utero. La placenta fue muestreada de acuerdo con las recomendaciones de lyengar y Rapp ${ }^{(10,20,21)}$. Las placentas expulsadas fueron recibidas en vasijas de acero inoxidable y se colocaron sobre gasas estériles para un secado, luego fue cortada en cuatro cuadrantes y de cada una se tomó 50 gramos de muestra, teniéndose $200 \mathrm{~g}$ de muestra por cada placenta que luego fueron almacenadas en bolsas estériles con cierre hermético y llevadas inmediatamente a la congeladora junto con las muestras hematológicas hasta completar el número total de muestras. Posteriormente, las muestras fueron descongeladas y secadas (seis horas en estufa a $70{ }^{\circ} \mathrm{C}$ ), finalmente fueron molidas y almacenadas en frascos de vidrio de $5 \mathrm{~mL}$, codificados y remitidos al Laboratorio de Química del IPEN para el análisis de Pb por espectrofotometría de absorción atómica con horno de grafito.

Para los análisis de Pb se utilizó un equipo de absorción atómica SHIMADZU AA-6800, empleándose 300 mg por muestra, la cual fue digerida con una mezcla de ácido nítrico y ácido perclórico de calidad ultrex, hasta sequedad, a fin de eliminar todos los compuestos orgánicos. Luego se retomó una muestra de $10 \mathrm{~mL}$ en una fiola, obteniendo una concentración al $20 \%$ en ácido. Se hicieron análisis por duplicado. En el caso del material de referencia los resultados responden al promedio de seis determinaciones 
de NRC-CNRC- DORM-2 es Dogfish Muscle. El uso del método de espectrofotometría de absorción atómica es aceptado por el Instituto Nacional de Seguridad e Higiene en el Trabajo (INSHT) y la OMS. Las concentraciones de Pb de la sangre materna y del cordón umbilical se expresaron el ug/dL, mientras que el de la placenta en ug/100 g.

El transporte transpalcentario de $\mathrm{Pb}$ materno al fetal, se calculó determinando la cantidad porcentual que representa el nivel de $\mathrm{Pb}$ en la sangre del cordón umbilical con respecto al contenido de $\mathrm{Pb}$ de la sangre materna que representaba el $100 \%$ de $\mathrm{Pb}$, siguiendo las sugerencias de diferentes autores ${ }^{(6,8,11)}$.

\section{ANÁLISIS ESTADÍSTICO}

Se usó estadística descriptiva, determinándose las principales medidas de tendencia central y de dispersión. Para determinar diferencias entre el nivel de $\mathrm{Pb}$ de las madres y los neonatos se realizó una prueba de $t$ para diferencias de medias con datos pareados. Para establecer las asociaciones entre los contenidos de $\mathrm{Pb}$ en sangre materna, neonatal y placenta, se utilizaron diferentes modelos de regresión. Se calcularon los coeficientes de correlación de Pearson. Se elempleó el software SPSS, Minitab y Excel for Windows.

\section{ASPECTOS ETICOS}

En consideración a los derechos de las personas y de acuerdo a las normas bioéticas internacionales, se elaboró una hoja de consentimiento informado aprobado por el Instituto de Seguridad Alimentaria de la Universidad Nacional Agraria La Molina, la cual fue firmada voluntariamente por las madres participantes del estudio. A los 15 días post parto se visitó a las madres de familia en su domicilio, dándoles sugerencias de buenas prácticas alimentarias para sus hijos para contrarestar los efectos de la contaminación por $\mathrm{Pb}$.

\section{RESULTADOS}

\section{CARACTERISTICAS GENERALES}

Se analizaron 40 muestras de sangre materna, placenta y sangre del cordón umbilical ( $20 \%$ de los partos anuales de La Oroya). La edad promedio de las madres fue $29 \pm 6$ años. El promedio de años de residencia fue $16,5 \pm 10,6$. El promedio de partos, sin considerar el último parto, fue dos. La edad gestacional promedio al momento del parto fue de 39 semanas. El $52,5 \%$ de las madres vivían en La Oroya Nueva, el 22,5\% en La Oroya Vieja y el $25 \%$ restante en zonas más alejadas de la fundición, que durante el día laboraban en La Oroya Vieja y Nueva.
Tabla 1. Plomo en sangre de cordón umbilical y probables efectos sobre la salud en neonatos de La Oroya, Perú

\begin{tabular}{|c|c|c|}
\hline de plomo y efectos sobre la salud & $N=40$ & $(\%)$ \\
\hline $\begin{array}{l}<10 \text { ug/dL: Función de audición y crecimiento } \\
\text { disminuidos }\end{array}$ & 13 & $(32,5)$ \\
\hline $10-<20$ ug/dL: Coeficiente intelectual disminuido & 8 & $(20,0)$ \\
\hline $\begin{array}{l}\text { locidad de conducción } \\
\text { a }\end{array}$ & 12 & 30, \\
\hline $\begin{array}{l}30 \text { - }<40 \text { ug/dL: Metabolismo de la vitamina D } \\
\text { disminuido }\end{array}$ & 3 & $(7,5)$ \\
\hline $\begin{array}{l}\geq 40 \text { ug/dL: Producción de hemoglobina } \\
\text { disminuida }\end{array}$ & 4 & , \\
\hline
\end{tabular}

\section{PLOMO EN SANGRE MATERNA, PLACENTA Y CORDÓN UMBILICAL}

Los promedios de concentración de $\mathrm{Pb}$ en la sangre materna y cordon umbilical fueron $27,4 \pm 15,6 \mathrm{ug} / \mathrm{dL}$ y $19,0 \pm 12,6 \mathrm{ug} / \mathrm{dL}$, respectivamente $(\mathrm{p}<0,01)$. El promedio de $\mathrm{Pb}$ en la placenta fue $319,0 \pm 215,9 \mathrm{ug} / 100 \mathrm{~g}$. Los promedios de $\mathrm{Pb}$ en sangre materna para las madres de La Oroya Nueva y La Oroya Vieja fueron $24,2 \pm 16,8$ y $36,9 \pm 14,5 \mathrm{ug} / \mathrm{dL}(\mathrm{p}<0,01)$. Los promedios de $\mathrm{Pb}$ en cordon umbilical para los neonatos de Oroya Nueva y Vieja fueron $15,0 \pm 11,6$ y $22,6 \pm 9,4(p<0,01)$. El $32,5 \%$ de los neonatos tuvo menos de $10 \mathrm{ud} / \mathrm{dL}$ de $\mathrm{Pb}$, mientras que el $67,5 \%$ supero el nivel crítico. El $10 \%$ de los recién nacidos tuvo más de $40 \mathrm{ug} / \mathrm{dL}$ de $\mathrm{Pb}$. La concentración de $\mathrm{Pb}$ en el tejido placentario fue $285,4 \pm$ $251,4 \mathrm{ug} / 100 \mathrm{~g}$ para La Oroya Nueva y de 418,5 $\pm 126,4$ $\mathrm{ug} / 100 \mathrm{~g}$ para La Oroya Vieja $(p<0,01)$. Con respecto al transporte transplacentario, el $\mathrm{Pb}$ de cordón umbilical representó el $61,6 \%$ del $\mathrm{Pb}$ de la sangre materna.

En la Tabla 1 se muestran las frecuencias absolutas y relativas considerando los niveles de $\mathrm{Pb}$ en la sangre del neonato que ocasionan diferentes efectos sobre la salud, observándose que el $67,5 \%$ de los neonatos presentaron contenidos superiores a $10 \mathrm{ug} / \mathrm{dL}$.

Las curvas de regresión que mejor se ajustaron al evaluar el efecto del nivel de $\mathrm{Pb}$ en la sangre materna sobre el nivel de $\mathrm{Pb}$ en cordón umbilical fueron las cuadráticas. Las ecuaciones de regresión para los niveles de $\mathrm{Pb}$ de cordon umbilical respecto al $\mathrm{Pb}$ en sangre materna, $\mathrm{Pb}$ de la placenta respecto al $\mathrm{Pb}$ de la sangre materna y del $\mathrm{Pb}$ de cordon umbilical respecto al $\mathrm{Pb}$ de la placenta mostraron una correlación de Pearson de 0,36; 0,48 y 0,33 , respectivamente.

En las Tabla 2 y 3 se muestran los niveles de $\mathrm{Pb}$ en sangre materna y cordón umbilical con relación a la zona de residencia, observándose que todas de las madres residentes en la zona de fundición de La Oroya 
Tabla 2. Nivel de plomo en sangre materna con relación a la zona de residencia en La Oroya, Perú

\begin{tabular}{lcc}
\hline \multirow{2}{*}{ Zona de residencia } & \multicolumn{2}{c}{ Nivel de plomo en sangre materna } \\
\cline { 2 - 3 } & $<\mathbf{1 0}$ ug/dL (\%) & $\geq 10$ ug/dL (\%) \\
\hline La Oroya Nueva & $6(28,6)$ & $15(71,4)$ \\
La Oroya Vieja & $0(0,0)$ & $9(100,0)$ \\
Otras zonas & $2(17,5)$ & $8(82,5)$ \\
\hline
\end{tabular}

Tabla 3. Nivel de plomo en sangre de cordón umbilical con relación a la zona de residencia en La Oroya, Perú

\begin{tabular}{lcc}
\hline \multirow{2}{*}{ Zona de residencia } & \multicolumn{2}{c}{ Nivel de plomo en cordón umbilical } \\
\cline { 2 - 3 } & $<\mathbf{1 0} \mathbf{u g} / \mathbf{d L}(\%)$ & $\mathbf{1 0} \mathbf{u g} / \mathbf{d L}(\%)$ \\
\hline La Oroya Nueva & $10(47,6)$ & $11(52,4)$ \\
La Oroya Vieja & $1(11,1)$ & $8(88,9)$ \\
Otras zonas & $3(30,0)$ & $7(70,0)$ \\
\hline
\end{tabular}

Vieja tuvieron más de $10 \mathrm{ug} / \mathrm{dL}$ de $\mathrm{Pb}$; y en el caso de los neonatos, el $88,9 \%$ tuvieron más de $10 \mathrm{ug} / \mathrm{dL}$ de $\mathrm{Pb}$ en cordón umbilical.

\section{DISCUSIÓN}

De acuerdo con los resultados obtenidos en el presente estudio, las concentraciones sanguíneas de $\mathrm{Pb}$, tanto en sangre materna y del cordón umbilical están por encima del punto de corte (10ug/dL) establecido por el CDC y la OMS ${ }^{(16-18)}$, registrándose una concentración 1,52 veces más alta en las madres de La Oroya Vieja (zona de fundición), lo que demuestra que las gestantes de la ciudad de La Oroya tienen una exposición muy intensa a este metal pesado.

El promedio de $\mathrm{Pb}$ en sangre materna determinado en el presente estudio $(27,4 \mathrm{ug} / \mathrm{dL})$ fue mayor que el reportado en una zona de fundición de Yugoslavia, con $17,1 \mathrm{ug} / \mathrm{dL}$ (23); con respecto al contenido promedio de $\mathrm{Pb}$ en sangre de cordon umbilical, este fue hasta casi dos veces más alto que el límite crítico establecido para los neonatos ${ }^{(16-18)}$.

En zonas de baja contaminación, como en México, de 1404 partos, entre 1991 y 1993, se reportaron promedios de $\mathrm{Pb}$ en sangre materna y neonatal de $10,7 \pm 6,5$ y $10,4 \pm 6,2 \mathrm{ug} / \mathrm{dL}$, respectivamente ${ }^{(24)}$. En otro estudio realizado en Maracaibo, Venezuela, se evaluaron 70 partos, y se reportó concentraciones promedio de $\mathrm{Pb}$ de $6,6 \pm 3,3 \mathrm{ug} / \mathrm{dL}$ para sangre materna y $5,3 \pm 3,2 \mathrm{ug} / \mathrm{dL}$ para sangre neonatal, siendo el $\mathrm{Pb}$ en sangre neonatal el $80,2 \%$ del $\mathrm{Pb}$ materno ${ }^{(25)}$. Un estudio en California evaluó 723 nacimientos normales, y reportó un promedio de $5 \mathrm{ug} / \mathrm{dL}$ de $\mathrm{Pb}$ en sangre de cordón umbilical; el $3 \%$ de los neonatos excedieron de $10 \mathrm{ug} / \mathrm{dL}$ incrementándose a
$40 \%$ en prematuros ${ }^{(26)}$. Otro estudio en Braunschweig, Alemania ${ }^{(27)}$ en 9189 neonatos, reportó un promedio de $3,8 \pm 1,7 \mathrm{ug} / \mathrm{dL}$, donde el $4,7 \%$ tuvo más de $10 \mathrm{ug} / \mathrm{dL}$. En Australia (28), un estudio realizado en 51 mujeres, el promedio de $\mathrm{Pb}$ en la sangre materna y neonatal fue de $3,7 \pm 1,3$ y $2,6 \pm 1,6 \mathrm{ug} / \mathrm{dL}$, respectivamente. El $\mathrm{Pb}$ neonatal representó el $71,1 \%$ del $\mathrm{Pb}$ materno. En Szczecin, Polonia (11), en 83 partos, los niveles de $\mathrm{Pb}$ materno y neonatal fueron de 2,56 y $2,01 \mathrm{ug} / \mathrm{dL}$, respectivamente; el $\mathrm{Pb}$ neonatal representó el $78,5 \%$ del materno. En Estanbul, Turquía ${ }^{(8)}$, el promedio de $\mathrm{Pb}$ en sangre materna y neonatal fue de $2,4 \pm 0,9$ y $1,7 \pm$ $0,9 \mathrm{ug} / \mathrm{dL}$, respectivamente; siendo el $\mathrm{Pb}$ del neonato el $71,3 \%$ del $\mathrm{Pb}$ materno. Un estudio realizado en Bangkok ,Tailandia ${ }^{(29)}$, reporta niveles de $\mathrm{Pb}$ en la sangre materna y neonatal de $6,2 \pm 2,0$ y $1,6 \pm 0,5 \mathrm{ug} / \mathrm{dL}$, respectivamente; el $\mathrm{Pb}$ neonatal representó el $25,8 \%$ del $\mathrm{Pb}$ materno. En Shanghai, China en 348 neonatos se reportó un promedio de $\mathrm{Pb}$ de 9,2 ug/dL donde el 40,8\% tuvieron niveles superiores a $10 \mathrm{ug} / \mathrm{dL}{ }^{(30)}$.

Estudios realizados en áreas urbanas de mayor contaminación reportan mayores niveles de $\mathrm{Pb}$ materno y neonatal, entre ellos el realizado en Petras, Grecia, en 47 partos, el cual reporta promedios de $\mathrm{Pb}$ en la sangre materna y neonatal de $14,9 \pm 4,1$ y $13,1 \pm 3,7 \mathrm{ug} / \mathrm{dL}$, respectivamente; el $\mathrm{Pb}$ del neonato representó el $88 \%$ del $\mathrm{Pb}$ materno con una correlación altamente significativa $(r=0,413 ; p<0,01)^{(31)}$. Otro estudio en la zona urbana de Grecia ${ }^{(32)}$, reporta promedios de $\mathrm{Pb}$ en la sangre materna de $37,2 \pm 4,7 \mathrm{ng} / \mathrm{ml}$ y de $20 \pm 3,4 \mathrm{ng} / \mathrm{mL}$ en sangre neonatal.

Según nuestros resultados los recién nacidos tienen niveles altos de $\mathrm{Pb}$ en sangre, sobre todo en aquellos cuyas madres viven en zonas cercanas a la fundición. Este problema de salud pública debe ser enfocado integralmente con fines de reducir sus graves efectos sobre la salud de los recién nacidos; quienes, de continuar ingiriendo $\mathrm{Pb}$ por medio de la lactancia materna y por la via inhalatoria, $y$, posteriormente, mediante la ingesta de agua y alimentos, de reiniciar la fundición sus actividades en iguales condiciones que en la década pasada, sufrirán las consecuencias del envenenamiento crónico por este elemento tóxico. El Pb se irá almacenando en diversos órganos y tejidos, principalmente en el tejido óseo ${ }^{(33,34)}$, cerebro, hígado, riñones, tejido adiposo, entre otros; afectando principalmente la biosíntesis del grupo heme de la hemoglobina, haciendo más susceptibles a que los neonatos presenten cuadros de anemia; interferiendo además en el desarrollo del cerebro ${ }^{\left({ }^{34}\right)}$, ocasionando alteraciones neurofisiológicas, electroencefalográficas, defectos en la audición, disminución en la velocidad de conducción nerviosa, y si es que los niveles sobrepasan los $70 \mathrm{ug} / \mathrm{dL}$, la intoxicación puede provocar coma cerebral y muerte ${ }^{(36-39)}$; por lo que si la empresa 
metalúrgica tiene planificado reiniciar actividades metalúrgicas debe considerar juntamente con el MINSA y EsSalud las medidas de prevención a la exposición por $\mathrm{Pb}$, además de controlar rigurosamente las emisiones metalúrgicas.

Los promedios de $\mathrm{Pb}$ en la placenta determinados en este estudio, fueron más altos que los promedios de 48 a 76 ug/100g, reportados para una zona de fundición ${ }^{(40)}$, lo que indica que el nivel de contaminación permitía que la placenta concentre elevadas cantidades de $\mathrm{Pb}$ durante el embarazo, el cual atravesaría con facilidad hacia el feto. Es así que, a nivel de La Oroya Nueva y La Oroya Vieja los promedios de $\mathrm{Pb}$ en la placenta fueron 285,4 y 418,5 ug/100g.

Con respecto a las correlaciones determinadas en el presente estudio, es importante considerar que a partir del contenido de $\mathrm{Pb}$ en la placenta y en la sangre materna se puede estimar el contenido de $\mathrm{Pb}$ en la sangre del neonato, sin necesidad de recurrir a técnicas invasivas, puesto que la placenta es un residuo fetal que se elimina. Obviamente estas correlaciones son válidas para las condiciones en las que se realizó el presente estudio, es decir en zonas de altura y con presencia de altos niveles de contaminación por $\mathrm{Pb}$.

El estudio presenta algunas limitaciones; pues aun cuando se adoptan medidas de aseguramiento de la calidad, el almacenamiento y manipulación de las muestras en el hospital puede llevar a algún grado de contaminación, el cual debe estar exento de $\mathrm{Pb}$. Otro aspecto es que las madres del estudio han vivido en la zona del estudio entre seis a más de 30 años, ya sea en La Oroya Nueva o Vieja, afectando el nivel de deposición de $\mathrm{Pb}$ en el hueso materno, el cual durante la gestación va pasando al feto. En los casos de altos niveles de $\mathrm{Pb}$ en los neonatos no se realizó ninguna intevención de quelación.

Las conclusiones a las que se llegaron fueron las siguientes. La concentración de $\mathrm{Pb}$ en la sangre materna en las madres que viven en la zona de La Oroya Vieja fue 1,52 veces más alta que en el caso de las madres que viven en La Oroya Nueva. La cercanía a la fundición eleva la concentración sanguínea de $\mathrm{Pb}$. A mayor tiempo de residencia en La Oroya, la concentración sanguínea de $\mathrm{Pb}$ en la sangre de las gestantes es más alta, constituyéndose en un factor que influye sobre el nivel de contaminación en las gestantes y sus recién nacidos. La concentración de $\mathrm{Pb}$ en la sangre del cordón umbilical de los neonatos provenientes de madres que viven en La Oroya Vieja fue 1,51 veces más alta que de las provenientes de madres que viven en La Oroya Nueva. Asimismo, a mayor tiempo de residencia en La Oroya, mayor nivel de $\mathrm{Pb}$ en la sangre neonatal. La concentración de $\mathrm{Pb}$ en la placenta fue mayor en las mujeres que viven en La Oroya Vieja y en aquellas que radican un mayor tiempo en dichas condiciones. El Pb atravieza facilmente la barrera placentaria, siendo en promedio $69,5 \%$, con un rango de 61,3 a $94,7 \%$. Todas las madres que radican en La Oroya Vieja tuvieron más de $10 \mathrm{ug} / \mathrm{dL}$ de $\mathrm{Pb}$; mientras que en las de La Oroya Nueva el $71,4 \%$ sobrepasaron dicho nivel.

Agradecimientos: al Dr. Guillermo López de Romaña (Q.E.P.D.) por su asesoramiento y recomendaciones. A la Dra. Patricia Bedregal Salas, jefa del Laboratorio de Análisis del IPEN. AI Dr. Hugo Villa y el personal del Departamento de Obstetricia del Hospital II EsSalud de la Oroya. A las madres de familia que gentil y voluntariamente participaron del estudio.

Contribuciones de autoría: JCB y DCP participaron en la concepción, diseño del trabajo, recolección de las muestras, análisis e interpretación de datos y redacción del manuscrito. ERR participó en la fase de procesamiento de muestras para su envío al IPEN. Todos los autores participaron en la aprobación de la versión final.

Fuente de financiamiento: estudio cofinanciado por la Agencia Internacional de Energía Atómica (IAEA) Vienna, Austria quien tuvo a su cargo el $90 \%$ de los costos del estudio y el resto fue asumido por los investigadores.

Conflictos de interés: Los autores declaran no tener conflictos de interés.

\section{REFERENCIAS BIBLIOGRÁFICAS}

1. Carpenter DO. Effects of metals on the nervosu system of human and animals. Int J Occup Med Environ Health. 2001;14(3):209-18.

2. Gardella C. Lead exposure in pregnancy: a review of the literature and argument for routine prenatal screening. Obstet Gynecol Surv. 2001;56(4):231-8.

3. Sanborn MD, Abelsohn A, Campbell M, Weir E. Identifying and managing adverse environmental heatl effects: 3. Lead exposure. CMAJ. 2002;166(10):1287-92.

4. Wade MG, Parent S, Finnson KW, Foster W, Younglai E, McMahon A, et al. Thyroid toxicity due to subchronic exposure to a complex mixture of 16 organochlorines, lead, and cadmium. Toxicol Sci. 2002;67(2):207-18.

5. Carrión N. Determinación de metales pesados: Métodos de análisis en ambientes y sistemas biológicos. Caracas: Universidad Central de Venezuela; 2002.

6. Li PJ, Sheng YZ, Wang QY, Gy LY, Wang YL. Transfer of lead via placenta and breast milk in human. Biomed Environ Sci. 2000;13(2):85-9.

7. Osman K, Akesson A, Berglund M, Bremme K, Schutz A, Ask K, et al. Toxic and essential elements in placentas 
of Swedish women. Clin Biochem. 2000;33(2):131-8.

8. Furman A, Laleli M. Maternal and umbilical cord blood lead levels: an Istanbul study. Arch Environ Health. 2001; 56(1):26-8.

9. Horowitz BZ, Mirkin DB. Lead poisoning and chelation in a motherneonate pair. J. Toxicol Clin Toxicol. 2001;39(7):727-31.

10. Iyengar GV, Rapp A. Human placenta as a 'dual' biomarker for monitoring fetal and maternal environment with special reference to potentialy toxic trace elements. Part 2: Essential minor, trace and other (non-essential) elementns in human placenta. Sci Total Environ. 2001;280(1-3):207-19.

11. Durska G, Kozielec T, Karakiewicz B. [Evaluation of transplacental gradient for cadmium and lead]. Ginekol Pol. 2002;73(1):43-9. [Article in Polish]

12. Carpenter DO, Mahthews MR, Parsons PJ, Horl N. Long-term potentia- tion in the piriform cortex is blocked by lead. Cel Mol Neurobiol. 1994;14:723-33.

13. Winneke $G$, Lilienthal $H$, Krämer U. The neurobehavioural toxicology and teratology of lead. Arch Toxicol Suppl. 1996;18:57-70.

14. Rosen JF. Effects of low levels of lead exposure. Science 256. 1992; 256(5055):294.

15. Rosen JF. Health effects of lead at low exposure levels. Expert consensus and rationale for lowering the definition of childhood lead poisoning. 1992; 146(11):1278-81.

16. U.S. Department of Health and Human Services, Public Health Service, Centers for Disease Control. Preventing lead poisoning in young children. Atlanta, GA: CDC; 1991.

17. Intoxicación por plomo: de la detección a la prevención primaria. Sal Púb Méx. 1995;37(3):264-76.

18. World Health Organization (WHO). Niveles de efectos mínimos de plomo en niños y adultos. Geneva:,WHO; 1994.

19. United States Department of Health. Preventing Lead Poisoning in Young Children: CDC Public Statement. Washington, DC: United States Department of Health. 1991; October.

20. Iyengar GV, Rapp A. Human placenta as a 'dual' biomarker for monitoring fetal and maternal environment with special reference to potentialy toxic trace elements. Part 1: Physiology, function and sampling of placenta for elemental characterisation. Sci Total Environ. 2001;280(1-3):195-206.

21. Iyengar GV, Rapp A. Human placenta as a 'dual' biomarker for monitoring fetal and maternal environment with special reference to potentialy toxic trace elements. Part 3: Toxic trace elements in placenta and placenta as a biomarker for these elements. Sci Total Environ. 2001;280(1-3):221-38.

22. Blacksmith Institute. La Oroya Lead Pollution [Internet]. New York: Blacksmith Institute;c2013 [citado el 12 de febrero del 2013]. Disponible en: http://www.blacksmithinstitute.org/ projects/display/36

23. Graziano JH, Popovac D, Factor-Litvak P, Shrout P, Kline J, Murphy MJ, et al. Determinants of elevated blood lead during pregnancy in a population surrounding a lead smelter in Kosovo, Yugoslavia. Environ Health Perspect. 1990;89:95-100.

24. Navarrete-Espinosa J, Sanín-Aguirre LH, Escandón-Romero C, BenitezMartínez G, Olaiz-Fernández G, Hernández-Avila M. Niveles de plomo sanguíneo en madres y recién nacidos derechohabientes del Instituto Mexicano del Seguro Social. Salud Publica Mex. 2000;42(5):391-6.

25. Romero RA, Granadillo VA, Navarro JA, Rodriguez-Iturbe B, Pappaterra J, Pirela H. Placental transfer of lead in mother/newborn pairs of Maracaibo City (Venezuela). J Trace Electrolytes Health Dis. 1990;4(4):241-3.

26. Satin KP, Neutra RR, Guirguis G, Flessel P. Umbilical cord blood lead levels in California. Arch Environ Health. 1991;46 (3):167-73

27. Meyer J, Geuenich HH, Robra BP, Windorfer A. [Determinants of lead concentration in the umbilical cord blood of 9189 newborns of a birth cohort in the goverment district of Braunschweigl]. Zentralbl Hyg Umweltmed. 1992;192(6)522-33. [Article in German]

28. Plockinger B, Dada K, Meisinger V. [Lead, mercury and cadmium in newborn infants and their mothers]. Z Geburtshilfe Perinatol. 1993;197(2):104-7. [Article in German]

29. Phuapradit W, Jetsawangsri T, Chaturachinda K, Noinongyao $\mathrm{N}$. Maternal and umbilical cord blood lead levels in Ramathibodi Hospital, 1993. J Med Assoc Thai. 1994;77(7):386-72.

30. Shen XM, Yan CH, Guo D, Wu SM, Li RQ, Huang H, et al. Umbilical cord blood lead levels in Shanghai, China. Biomed Environ Sci. 1997; 10(1):38-46
31. Nashashibi N, Cardamakis E, Bolbos G, Tzingounis $\mathrm{V}$. Investigation of kinetic of lead during pregnancy and lactation. Gynecol Obstet Invest. 1999;48(3):158-62

32. Dussias V, Stefos T, Stefanidis K, Paraskevaidis E, Karabini F, Lolis D. Lead concentrations in maternal and umbilical cord blood in areas with high and low air pollution. Clin Exp Obstet Gynecol. 1997;24(4):187-9.

33. González-Cossío T, Peterson KE, Sanín LH, Fishbein E, Palazuelos E, Aro A, et al. Decrease in birth weigth in relation to maternal bone-lead burden. Pediatrics. 1997;100(5):856-62.

34. Sanin LH, Gonzáles-Cossío T, Romieu I, Peterson KE, Ruiz S, Palazuelos E, et al. Effect of maternal lead burden on infant weigth and weigth gain at one month of age among breastfed infants. Pediatics. 2001;107(5):1016-23.

35. Winneke G, Lilienthal $H$, Krämer U. The neurobehavioural toxicology and teratology of lead. Arch Toxicol Suppl. 1996; 18:57-70.

36. Otto D, Benignus V, Muller K, Barton C, Seiple K, Prah J, et al. Effects of low to moderate lead exposure on slow cortical potentials in young children: two-year follow-up study. Neurobehav Toxicol Teratol. 1982;4(6):733-7

37. Schwartz J, Otto D. Blood lead levels, hearing thersholds, and neurobehavioral development in NHANES II children and youth. Cincinnati: Health Effects Research Laboratory, United States Environmental Protection Agency (USEPA); 1986.

38. Seppäläinen AM, Hernberg S. A followup study of nerve conduction velocities in lead exposed workers. Neurobehav Toxicol Teratol. 1982;4(6):721-3.

39. Calderón-Salinas JV, Hernandez-Luna C, Valdez-Anaya B, Maldonado-Vega M, Lopez-Miranda A. Evolution of lead toxicity in a population of children. Hum Exp Toxicol. 1996;15(5):376-82.

40. Baghurst PA, Robertson EF, Oldfield RK, King BM, McMichael AJ, Vimpani GV, et al. Lead in the placenta, membranes, and umbilical cord in relation to pregnancy outcome in a leadsmelter community. Environ Health Perspect. 1991;90:315-20.

Correspondencia: Jorge Castro Bedriñana.

Dirección: Jr. A.B. Leguía 110-Chilca, Huancayo, Perú

Teléfono: (064) 223371

Correoelectrónico:jorgecastrobe@yahoo.com 